\title{
Educación emocional en la escuela. Una revisión de la literatura
}

Elena Pastor-Porras - Universidad de Valencia Diana Marín Suelves - Universidad de Valencia

Recepción: 28.09.2021 | Aceptado: 19.10.2021

Correspondencia a través de ORCID: Diana Marín Suelves
0000-0003-2024-5235

0000-0002-5346-8665

Citar: Pastor-Porras, E y Marín Suelves, D (2021). Educación emocional en la escuela. Una revisión de la literatura. REIDOCREA,1-19.

Área del conocimiento: Educación

Resumen: El presente artículo muestra una revisión de la literatura a través de la cual se pretende buscar evidencias para resaltar la Educación Emocional como una herramienta para resolver los conflictos y dificultades surgidos en el ámbito de la escuela ordinaria. Para buscar y seleccionar los documentos analizados, se han empleado las bases de datos Scopus, Web of Science y Dialnet, teniendo en cuenta unos criterios de inclusión y exclusión. De esta manera, se han examinado un total de 21 estudios. En general, en la mayoría de ellos se demuestra el incremento y/o la mejora de las competencias emociones $\mathrm{y}$, por consiguiente, el desarrollo de habilidades para el mantenimiento de relaciones positivas y, en diversas circunstancias, una mejora del rendimiento escolar, comportando un beneficio para el clima del aula. Tomando en consideración los resultados obtenidos, se hace evidente la necesidad de incorporar la Educación Emocional en el currículo académico de Educación Primaria y Secundaria para favorecer el desarrollo integral (desarrollo de la personalidad, progreso académico y vida social) de los niños y niñas.

Palabra clave: Educación Emocional

\section{Emotional education at school. A review of the literature}

Abstract: This article presents a literature review through which it is intended to search for some evidence to highlight Emotional Education as a tool to resolve conflicts and difficulties arising in the field of ordinary school. In order to search and select the analyzed documents, the Scopus, Web of Science and Dialnet databases have been used, considering inclusion and exclusion criteria. In this way, a total of 21 studies have been examined. In general, most of them demonstrate the increase and/or improvement of emotional competencies and consequently the development of skills to maintain positive relationships and an improvement in school performance in various circumstances, involving a benefit for the classroom climate. Taking into consideration the results obtained, it is highlighted the need to incorporate Emotional Education in the academic curriculum of Primary and Secondary Education to promote the integral development (personality development, academic progress, and social life) of children.

Keyword: Emotional Education

\section{Introducción}

Tal y como afirma Bisquerra (2003) un porcentaje considerable de los jóvenes se implica en conductas de riesgo que conllevan un desequilibrio emocional, lo cual justifica la necesidad de diseñar intervenciones psicoeducativas, desde un enfoque preventivo, para la adquisición de competencias básicas para la vida (BOE, 2015, Art. 2).

Estas intervenciones deben incorporar herramientas y estrategias para la prevención y resolución de conflictos que dificultan la convivencia escolar y que acaban disminuyendo el rendimiento escolar y aumentando las tasas del suicidio juvenil, la depresión infantil y las conductas de riesgo (Vivas, 2003). De este modo, surge la necesidad de considerar los aspectos emocionales en la escuela (Goleman, 1995; Salovey y Mayer, 1997; BarOn, 2000), así como se atiende a la educación académica (García, 2012). 
A la hora de conceptualizar la educación emocional, se pueden identificar una amplia variedad de acepciones. Así, Bisquerra (2006) concibe la educación emocional como un proceso educativo, continuo y permanente, que procura favorecer el incremento de las competencias emocionales como componente fundamental del desarrollo global de la persona, con la finalidad de habilitarle para la vida y aumentar su bienestar personal y social. Por su parte, Cepa, Heras y Fernández-Hawrylak (2017) establecen que dicha educación plantea sugerencias para que el alumnado adquiera capacidades y aptitudes sobre las emociones para que, en un futuro, sea capaz de valorar las propias emociones y las de los demás, y de alcanzar un grado de competencia en la regulación de las mismas y en la toma de decisiones. Además, Bisquerra y Pérez-Escoda (2007) exponen que el propósito principal de la educación emocional es el desarrollo de competencias emocionales y el bienestar, que son el conjunto de conocimientos, capacidades, habilidades y actitudes imprescindibles para entender, exteriorizar y regular de forma adecuada los fenómenos emocionales (Pérez y Filella, 2019).

Son varios los autores que señalan la importancia de educar emocionalmente a los jóvenes para ayudarlos a regular las emociones que provocan los conflictos y enfrentamientos en la escuela (Bisquerra y Pérez Escoda, 2007, 2012; Vivas, 2003). Pues, tal y como indican Bisquerra y Pérez (2012), la adquisición de competencias emocionales fomenta las relaciones sociales e interpersonales, facilita la resolución de conflictos, beneficia la salud física y mental y contribuye a mejorar el rendimiento académico.

Teniendo en cuenta estas cuestiones, en este artículo se realiza una revisión bibliográfica de los estudios publicados sobre el impacto de la educación emocional y los conflictos en la escuela. En base a ellos se analiza el papel que la educación emocional como herramienta para la resolución de conflictos y problemas cotidianos desde un enfoque preventivo y rehabilitador. Por tanto, las preguntas que se pretende responder con este estudio son: ¿qué beneficios tiene educar emocionalmente a los alumnos? y ¿es una estrategia eficaz para evitar y/o resolver los conflictos que se desarrollan en las escuelas?

\section{Metodología}

Para la consecución de los objetivos se realizó una revisión de la literatura con el fin de analizar las publicaciones científicas sobre educación emocional y resolución de conflictos. Baena (2017), define la investigación documental como un procedimiento que trata de seleccionar y recopilar la información a través de la lectura y evaluación crítica de documentación y material bibliográfico. Se utilizaron tres bases de datos de reconocido prestigio: Scopus, Web of Science (WOS) y Dialnet. Las palabras clave empleadas, combinadas con los operadores booleanos OR/AND, en inglés y castellano fueron: intervention AND emotional education AND school OR.

Los criterios de inclusión y exclusión utilizados se muestran en la Tabla 1.

TABLA 1. CRITERIOS APLICADOS PARA LA SELECCIÓN DE ARTÍCULOS.

\begin{tabular}{l|l}
\hline \multirow{3}{*}{ INCLUSIÓN } & \multicolumn{1}{c}{ Critículos ciontíficos de intervención. } \\
& Publicados entre el año 2011 hasta la actualidad (2021), debido a que se considera un margen de rastreo \\
conveniente para identificar una gran cantidad de informes y evitar su desfase. \\
Divulgado en bases de datos de renombre, como Scopus, Dialnet y WOS (Web of Science). \\
Redactados en castellano, catalán o inglés. \\
De disponibilidad inmediata (acceso abierto). \\
Muestra que incluya individuos que pertenezcan a la escuela ordinaria.
\end{tabular}


Participantes escolarizados en Educación Primaria y Secundaria (de 5/6 a 16 años), es decir, el periodo de escolarización obligatoria.

Intervenciones que pongan a prueba programas de educación emocional o similares para observar resultados.

EXCLUSIÓN Artículos que se han repetido en las bases de datos mencionadas (WOS, Scopus y dialnet).

De accesibilidad restringida o comprendidos en bases de datos de pago.

Intervenciones llevadas a cabo anteriormente del periodo establecido (2011).

Documentos eliminados o no disponibles.

Intervención de individuos cuyas edades sean inferiores o superiores a la etapa de escolarización obligatoria

(5/6-16 años).

Intervención que no se haya realizado en la escuela.

Artículos en los que no se observe los resultados de un programa de intervención basado en la educación emocional.

Documentos de revisión bibliográfica o similares.

En la Figura 1 se muestra el proceso seguido en base a las orientaciones del método PRISMA (Urrútia y Bonfill, 2010).

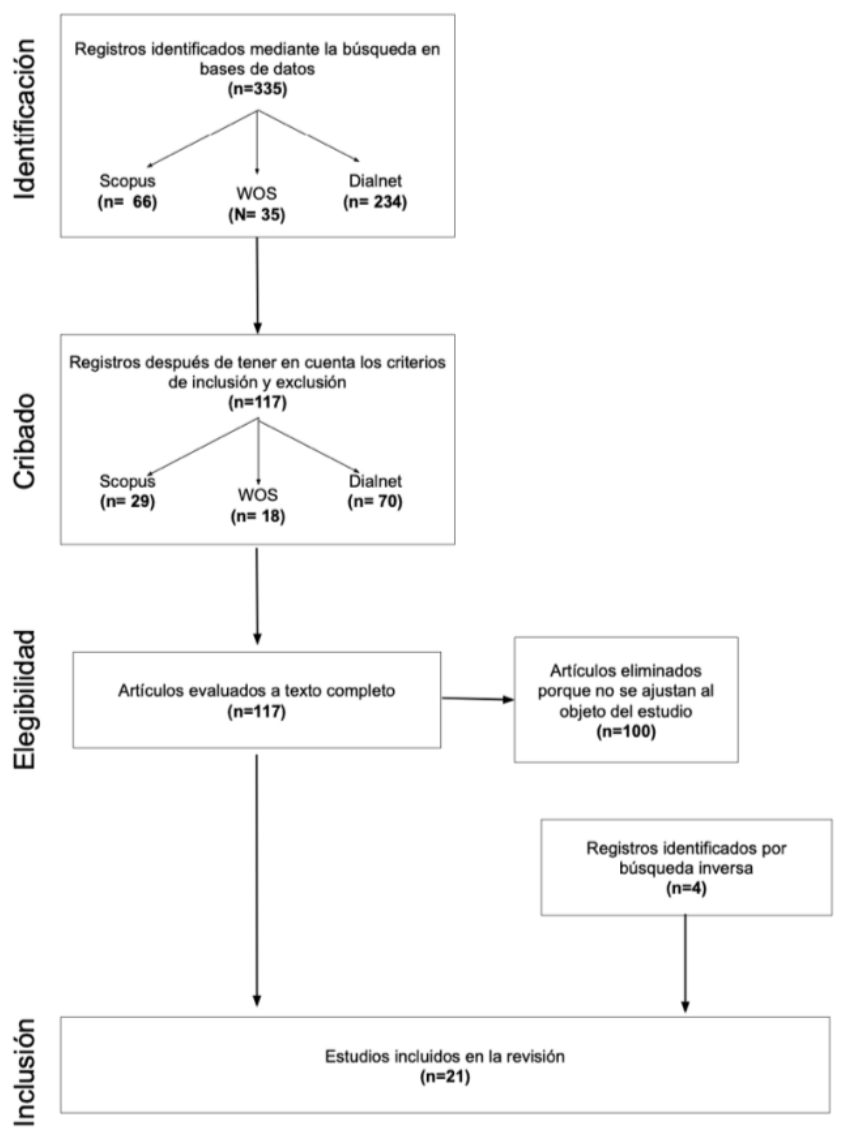

Figura 1. Diagrama de flujo del proceso de selección de artículos. 


\section{Resultados}

En la Tabla 2 se muestra la información clave de los 21 artículos que se han analizado incluyendo: autores y año de publicación, objetivos, muestra, tipo de estudio e instrumentos de medida, descripción de la intervención y resultados.

\begin{tabular}{|c|c|c|c|c|c|}
\hline AUTORES Y AÑO & Objetivos & $\begin{array}{l}\text { Descripción de } \\
\text { la muestra }\end{array}$ & $\begin{array}{l}\text { Tipo de estudio e instrumentos de } \\
\text { medida }\end{array}$ & Descripción de la intervención & Resultados \\
\hline $\begin{array}{l}\text { TUR-PORCAR, } \\
\text { LLORCA-MESTRE Y } \\
\text { MESTRE- ESCRIVÁ } \\
\text { (2021) }\end{array}$ & $\begin{array}{l}\text { Examinar los efectos del programa de } \\
\text { educación emocional y observar las } \\
\text { consecuencias en la conducta } \\
\text { agresiva y la inestabilidad emocional } \\
\text { de los alumnos }\end{array}$ & $\begin{array}{l}\mathrm{n}=555 \\
\text { Edad/curso= } \\
\text { Educación } \\
\text { Primaria, } 7-12 \\
\text { años } \\
\mathrm{GC}=317 \\
\mathrm{GE}=238\end{array}$ & $\begin{array}{l}\text { Investigación evaluativa cuasi- } \\
\text { experimental con grupo experimental y } \\
\text { control. } \\
\text { Instrumentos de medida: } \\
\text {-Escala de Agresividad Física y Verbal. } \\
\text {-Escala de Inestabilidad Emocional. } \\
\text { - Mann-Whitney U Test. } \\
\text { - Prueba de dignos de Wilcoxon Signed } \\
\text { Rank Test (WSRT) }\end{array}$ & $\begin{array}{l}\text { Programa para la intervención socioemocional. Se } \\
\text { ha desarrollado en } 16 \text { sesiones de dos horas de } \\
\text { duración. } \\
\text { Comprende competencias cognitivas, afectivas y } \\
\text { conductuales interconectadas, que se consideran } \\
\text { importantes para la autonomía y el éxito en la } \\
\text { escuela: autoconciencia emocional, autocontrol } \\
\text { emocional, habilidades de comunicación y relación } \\
\text { social, conciencia social y resolución de conflictos y } \\
\text { toma de decisiones. }\end{array}$ & $\begin{array}{l}\text { Los resultados muestran la eficacia del } \\
\text { programa al comprobarse la disminución de } \\
\text { la agresividad y la inestabilidad emocional. }\end{array}$ \\
\hline $\begin{array}{l}\text { AGUILAR, GARCÍA Y } \\
\text { GIL (2021) }\end{array}$ & $\begin{array}{l}\text { Comprobar y evaluar una intervención } \\
\text { para mejorar los vínculos afectivos en } \\
\text { los estudiantes, reconocer las } \\
\text { diferentes emociones en el aula como } \\
\text { prevención del acoso escolar, } \\
\text { favorecer el desarrollo de la } \\
\text { autoestima y fomentar el desarrollo de } \\
\text { la empatía en un clima de aula } \\
\text { positivo. }\end{array}$ & $\begin{array}{l}n=58 \\
\text { Edad= } 10-12 \\
\text { años } \\
\text { Lugar= un centro } \\
\text { escolar de } \\
\text { Córdoba. }\end{array}$ & $\begin{array}{l}\text { Estudio longitudinal, "ex post facto", } \\
\text { cuasi experimental, prepost de tres } \\
\text { grupos, con un grupo experimental. } \\
\text { Instrumentos de medida: } \\
\text {-Cuestionario Sociométrico. } \\
\text {-Cuestionario de relaciones } \\
\text { socioafectivas. }\end{array}$ & $\begin{array}{l}\text { Experiencia educativa llevada a cabo en Educación } \\
\text { Fisica, dentro del área de Expresión Corporal (EC). } \\
\text { Se ha desarrollado en } 12 \text { sesiones: } 3 \text { dirigidas a } \\
\text { familiarizar al alumnado con la rama de la EC; } 7 \text { se } \\
\text { centraron en la regulación motriz y emocional; las } \\
\text { últimas } 2 \text { sesiones se destinaron a la evaluación del } \\
\text { propio alumnado y a la reflexión de la experiencia. }\end{array}$ & $\begin{array}{l}\text { Beneficioso para el desarrollo de las } \\
\text { habilidades socioemocionales del } \\
\text { alumnado. } \\
\text { Mejoran las dimensiones de relación social, } \\
\text { respeto-responsabilidad y victimización- } \\
\text { agresión. } \\
\text { No se demuestra el fomento de la } \\
\text { autoestima de los escolares. No se } \\
\text { identifica la mejora de la asertividad o la } \\
\text { cooperación y liderazgo. } \\
\text { Los datos evidencian que el desarrollo de la } \\
\text { competencia social evita la implicación en } \\
\text { problemas de violencia interpersonal. } \\
\text { Clima de aula más positivo. }\end{array}$ \\
\hline $\begin{array}{l}\text { MARTÍNEZ-ÁLVAREZ, } \\
\text { HIDALGO-FUENTES, } \\
\text { SOSPEDRA- } \\
\text { BAEZA (2020) }\end{array}$ & $\begin{array}{l}\text { Potenciar la inteligencia emocional } \\
\text { para proporcionar al estudiantado } \\
\text { competencias socioemocionales, que } \\
\text { le permitan: incrementar su bienestar } \\
\text { personal, convertirse en personas } \\
\text { responsables, comprometidas y } \\
\text { cooperadoras, mejorar su calidad de } \\
\text { vida y aumentar sus logros } \\
\text { profesionales en el futuro. }\end{array}$ & $\begin{array}{l}n=198(103 \\
\text { chicos } / 95 \\
\text { chicas }) \\
\text { Edad/curso }=1^{\circ}-6^{\circ} \\
\text { primaria, } 6-12 \\
\text { años. } \\
\text { Lugar= un centro } \\
\text { educativo } \\
\text { concertado de } \\
\text { Tolosa }\end{array}$ & $\begin{array}{l}\text { Estudio descriptivo, comparativo y } \\
\text { correlacional. } \\
\text { Instrumentos de medida: } \\
\text {-Batería de Adaptación Social (BAS-1) }\end{array}$ & $\begin{array}{l}\text { Para el desarrollo del programa, se han seguido } \\
\text { unos principios que se basan en una metodología } \\
\text { lúdica y participativa, y en la que se ha implicado a } \\
\text { la totalidad de la comunidad educativa. } \\
\text { Las actividades se organizan en } 5 \text { bloques de } \\
\text { contenido (competencias socioemocionales): la } \\
\text { conciencia emocional, la regulación emocional, la } \\
\text { autonomía personal, las habilidades sociales y las } \\
\text { habilidades de vida y bienestar. }\end{array}$ & $\begin{array}{l}\text { Desarrollo de las competencias } \\
\text { socioemocionales y mejora de los factores } \\
\text { facilitadores de la socialización. Es decir, se } \\
\text { facilita el desarrollo de aspectos } \\
\text { relacionados con la adaptación social. } \\
\text { La puntuación global en socialización } \\
\text { (mayor inteligencia emocional) es } \\
\text { significativamente mayor en las chicas que } \\
\text { en los chicos. } \\
\text { Diferencias positivas en el rendimiento } \\
\text { académico del grupo. }\end{array}$ \\
\hline
\end{tabular}




ECHEVERRÍA, LÓPEZ-
LARROSA Y MENDIRI
(2020)
SIGÜEÑA-MARIN,
CARBALLIDO-
GUISADDO, PÉREZ-
ALBÉNIZ Y FONSECA-
PEDRERO (2019)
POSTIGO-ZEGARRA,
SCHOEPS,
MONTOYA-CASTILLA
Y ESCARTI (2019)

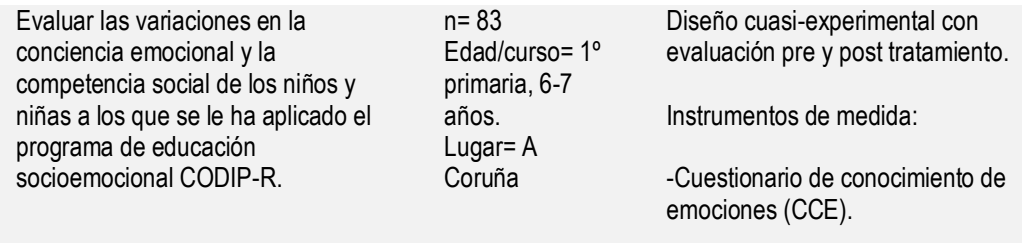

Implementar un programa de educacion emocional con el objetivo de desarrollar, promover y/o potenciar las competencias socioemocionales. Analizar los beneficios del programa sobre diversos indicadores de salud mental, autoestima, empatía e inteligencia emocional, y analizar satisfacción de los usuarios.

Evaluar los efectos percibidos en el Programa de Educación Emocional para adolescentes (PREDEMA) sobre la inteligencia emocional, los afectos positivos y negativos, las conductas de bullying y cyberbullying.

$n=104 \quad$ Diseño longitudinal de tipo cuasiESO, 13-14

años. tratamiento.

Lugar= diferentes Instrumentos de medida:

centros

ducativos de La -Cuestionario de Capacidades y Quiestionnaire, SDQ) control= 44 Mood Scale- 24, TMMS-24) - - Euestionario de satisfacción con el $\mathrm{n}=293$ (156 Diseño mixto que integra datos chicos/ 137 chicas) Edad $/$ curso $=1^{\circ} \mathrm{y}$ $2^{\circ}$ ESO, $11-15$ años
El programa se ha puesto en marcha durante un curso académico completo, con sesiones

semanales.

Se ha partido de Children of Divorce Intervention Program (CODIP) y se emplea la versión en castellano CODIP-R.

CODIP es un programa de intervención y

prevención centrado en el juego no competitivo. S cuenta con la presencia de una mascota. Promueve la adquisición de habilidades unidas a la expresion e identificación de emociones y la resolución practica de conflictos.

Se han llevado a cabo 6 sesiones en el centro escolar.

El programa de intervención se basa en el Edad/curso $=\quad$ experimental con evaluación pre y post Rioja. Dificultades (Strengths and Difficulties

$\mathrm{GE}=60$ Grupo de -La escala Rasgo de Metaconocimiento sobre Estados Emocionales-24 (Trat -Escala de Autoestima de Rosenberg. - Cuestionario de satisfacción con el programa INTEMO, con algunas modificaciones se ha renombrado como INTEMO-UR.

Se han organizado las sesiones en cuatro fases, que se corresponden con las cuatro ramas del modelo de IE de Mayer y Salovey (1997).

La metodología utilizada ha sido lúdica, atractiva, vivencial práctica grupal, dinámica y participativa. Además pre han tenido en cuenta los intereses del Además, se han tenido en cuenta los intereses del través de diferentes dinámicas y juegos. Se han desarrollado 8 sesiones, 1 a la semana.

Programa de Educación Emocional para Adolescentes (PREDEMA) se basa en el modelo de Mayer y Salovey (1997), y su objetivo es desarrollar las cuatro competencias emocionales que plantea. Ha constado de 11 sesiones de 50 minutos. En todas ellas se ha utilizado la misma secuencia de trabajo, basado en una manera natural de aprender experiencias personales o interpersonales donde las emocionales son significativas, elaboración de teorías o significados personales sobre esas experiencias, etc. cuantitativos.

Instrumentos de medida:

- Cyberbullying: Screening de acoso entre iguales.

-Trait Meta-Mood Scale (TMMS-24).

Scale of Positive and Negative

Experiences (SPANE).
Aumento de la conciencia emocional autoconciencia emocional y capacidad de cher gestín positia de los conflics

interpersonales) del alumnado participante.

\section{Problemas emocionales y}

comportamentales. No se hallaron

resultados estadísticamente significativos.

Cierta mejoría en la dimensión de

Problemas emocionales.

Autoestima, empatia e inteligencia emocional. Mejora de algunos aspectos relevantes de la IE, aunque no son

\section{significativos.}

Satisfacción de los usuarios. La mayoría de ellos muestran niveles elevados de satisfacción.

Emocional: Con respeto a la valoración, percepción y expresión emocional, se mejora la atencion y conciencia emocional (identificar y hablar de las emociones). Mejora de las competencias básicas (capacidad de regular las emociones desagradables) No se encuentran diferencias en la "claridad emocional". Social: Desarrollo de conductas prosociales de ayuda y apoyo hacia los compaños. de ayuda y apoyo hacia los companeros. No se victimización y observación del bullying. Persocion Personal: Mayor nivel de autodirección (conocimiento y aceptación de sí mismos, autoestima y autocontrol). Incremento en los "afectos positivos". 
PASTOR-ARNAU (2019)

CABELLO-CUENCA PEREZ-ESCODA, FILELLA-GUIU (2019)

SCHOEPS

VILLANUEVA, PRADOGASCÓ Y MONTOYACASTILLA (2018)
Integrar la educación emocional en el currículo de Educación Primaria desarrollar las competencias

emocionales del alumnado y optimizar el aprendizaje.

Analizar los efectos (con respecto a la gestión de las competencias emocionales) del programa Educación Emocional Happy 8-12 y de Happy 12-16.

Analizar el impacto de un programa de educación emocional para promover la convivencia y el bienesta en el aula mediante el desarrollo de competencias emocionales. $\mathrm{n}=88$ alumnos $\mathrm{dad} / \mathrm{curs} \mathrm{s}=4^{\circ}$ primaria, 9-10 años. Lugar $=4$ centros educativos alencianos.

$\mathrm{n}=574(301$

chicos/ 273

Edad/curso $=5^{\circ} \mathrm{y}$

$6^{\circ}$ de primaria

10-11 años.

$n=903$ chicos $y$

$\mathrm{Edad} / \mathrm{curso}=1^{\circ}$
$2^{\circ} \mathrm{ESO}, 12-13$

ños.

$n=148(64$

chicos/ 84

chicas)

Edad/curso $=1^{\circ} \mathrm{y}$

$2^{\circ} \mathrm{ESO}, 12-15$

años.

Lugar $=4$

escuelas

diferentes en la
Investigación evaluativa cuasi-

Programa PEEM, organizado de acuerdo con el

modelo pentagonal de Bisquerra (2009) y del control.

Instrumentos de medida:

-Cuestionario de Desarrollo Emociona (QDE 9-13)

-Escala de autoconcepto de Piers-

Harris (Piers, 1984)

-Se han tenido en cuenta las

calificaciones escolares y se han

utilizado instrumentos de recogida de

información como la entrevista con los

maestros, la observación sistemática

las valoraciones de las actividades por

parte del alumnado.

Diseño cuasi-experimental con pretest

y postest con un grupo de control.

nstrumentos de medida:

Alumnado de primaria:

-Cuestionario de Desarrollo Emociona (CDE).

-Stait-Trait Anxiety Inventory for

Children (STAIC)

Califcaciones de las asignaturas

instrumentales.

Alumnado de ESO:

-Cuestionario de desarrollo emocional

Secundaria (CDE SEC).

-Stait-Trait Anxiety Inventory (STAI).

-Calificaciones (notas medias) de todas

las asignaturas.

Diseño cuasi-experimental con una

intervención (experimental) y un grupo

de control.

Instrumentos de medida:

-Emotional Skills and Competencies Questionnaire (ESCQ).

-La cibervictimización a través de la

escala de victimización vía teléfon

móvil e internet (CYB-VIC).
GROP, y las corrientes pedagogicomusicales del

siglo XX.

Es un programa de educación emocional a través de la música, dentro del currículo.

Está formado por una selección de actividades

musicales caracterizadas por ser colectivas

participativas, prácticas y vivenciales. Está

der derion

del desarrollo emocional y los recursos

metodológicos musicales.

Se han llevado a cabo 2 sesiones semanales

durante 1 cuatrimestre.

Programas de Intervención Happy 8-12 y Happy 12-

Se trata de videojuegos diseñados para aprender a gestionar las competencias emocionales de niños adolescentes y, así, poder responder a los

conflictos de manera asertiva.

Cada uno presenta un total 25 conflictos orientados cumplir los objetivos descritos, al mismo tiempo que se trabajan las distintas competencias mocionales 15 de los conflictos se llevan a cabo en el contexto escolar y 10 entre hermanos, en contexto familiar.

Programa de intervención socioemocional

PREDEMA. Diseñado para promover la convivencia del aula y el bienestar subjetivo mediante el desarrollo de competencias emocionales.

Se ha llevado a cabo en once sesiones, en seis clases, por un psicólogo capacitado.

La primera parte, se ha centrado en las habilidade emocionales más básicas.

La segunda parte, centrada en la regulación y manejo emocional.
Mejora del autoconcepto y el rendimiento

as estrategias planteadas han promovido la actividad y la comunicación entre iguales y el docente.

En general, mejora del clima del aula.
Happy 8-12: mejora de las competencias mocionales.

Aunque no se ha observado una mejora significativa, si se refleja un aprendizaje discreto de todas las variables

emocionales.

Disminución notable de la ansiedad-estado. Mejora significativamente el rendimiento académico.

Happy 12-16. Efecto significativo en la competencia de Conciencia Emocional. ncremento significativo en las

competencias de vida y la autonomía

emocional.

Niveles de ansiedad-estado sin mejoría y aumentan.

El rendimiento académico mejora

significativamente.

No ha sido efectivo en el desarrollo de

competencias emocionales. Sin embargo,

mejora, en el seguimiento del programa, de

la capacidad para percibir, comprender y

regular las emociones.

Eficaz para promover la convivencia en el

aula al reducir la incidencia del

ciberacoso. Menos conductas

amenazadoras y humillantes a través de

dispositivos electronicos (una vez finalizado y también al transcurrir un tiempo). 


\begin{tabular}{|c|c|c|c|}
\hline \multirow[b]{2}{*}{$\begin{array}{l}\text { GÁLVEZ-IÑIGUEZ } \\
(2018)\end{array}$} & & & -Satisfaction With Life Scale (SWLS) \\
\hline & $\begin{array}{l}\text { Evaluar la efectividad de una } \\
\text { intervención de educación emocional, } \\
\text { para favorecer la adquisición de } \\
\text { habilidades sociales y de gestión } \\
\text { emocional para, posteriormente, } \\
\text { generalizarla. }\end{array}$ & $\begin{array}{l}\mathrm{n}=123 \\
\text { Edad/curso }=3^{\circ} \text { y } \\
4^{\circ} \text { de Educación } \\
\text { Primaria, } 8-9 \\
\text { años } \\
\text { Lugar= un centro } \\
\text { educativo privado } \\
\text { de la Comunidad } \\
\text { Valenciana. } \\
\mathrm{GC}=42 \text { alumnos. } \\
\mathrm{GE}=81 \text { alumnos. }\end{array}$ & $\begin{array}{l}\text { Diseño cuasi-experimental con } \\
\text { evaluaciones protest-postest con un } \\
\text { grupo control. } \\
\text { Instrumentos de medida: } \\
\text {-Escala Matson para la Evaluación de } \\
\text { Habilidades Sociales en Jóvenes } \\
\text { (MESSY). } \\
\text {-Escala de Autoestima de Rosenberg. } \\
\text {-Cuestionario de Conciencia Emocional } \\
\text { (EAQ-30). } \\
\text {-Cuestionario sociodemográfico con } \\
\text { preguntas ad hoc. } \\
\text {-Family Affluence Scale (FAS). } \\
\text {-ítems para evaluar el efecto de la } \\
\text { contaminación entre las condiciones } \\
\text { experimentales. }\end{array}$ \\
\hline $\begin{array}{l}\text { GARCíA Y ANDRÉS- } \\
\text { ROQUETA (2017) }\end{array}$ & $\begin{array}{l}\text { Valorar el efecto de una intervención } \\
\text { en educación emocional para mejorar } \\
\text { los problemas de conducta } \\
\text { detectados. }\end{array}$ & $\begin{array}{l}\mathrm{n}=24 \text { (55 chicas/ } \\
68 \text { chicos) } \\
\text { Edad/curso }=1^{0} \\
\text { de Educación } \\
\text { Primaria, } 6-8 \\
\text { años. } \\
\text { Lugar= Punta } \\
\text { Cana. }\end{array}$ & $\begin{array}{l}\text { Diseño cuasi-experimental de medidas } \\
\text { repetidas pretest-postest. } \\
\qquad \text { Instrumentos de medida: } \\
\text {-Aplicación Emocionatest. }\end{array}$ \\
\hline CEJUDO (2017) & $\begin{array}{l}\text { Diseñar, desarrollar y evaluar los } \\
\text { efectos de un programa para la } \\
\text { mejora de la inteligencia emocional } \\
\text { (PMIE) en el ajuste psicosocial y el } \\
\text { rendimiento académico. }\end{array}$ & $\begin{array}{l}\mathrm{n}=123 \\
\text { Edad/curso }=6^{0} \\
\text { de Educación } \\
\text { Primaria, 10-12 } \\
\text { años. } \\
\mathrm{GC}=55 \\
\mathrm{GE}=68\end{array}$ & $\begin{array}{l}\text { Diseño cuasi-experimental de medidas } \\
\text { repetidas pretest-postest con grupo de } \\
\text { control. } \\
\text { Instrumentos de medida: } \\
\text {-Indicador del rendimiento académico } \\
\text { global (RAG). } \\
\text { - Cuestionario abreviado de inteligencia } \\
\text { emocional como rasgo para } \\
\text { adolescentes (TEIQue-ASF). } \\
\text { - Sistema de evaluación de la conducta } \\
\text { de niños y adolescentes (BASC) } \\
\text { - Escala de afecto positivo y negativo } \\
\text { en su versión para niños (PANASN). }\end{array}$ \\
\hline
\end{tabular}

Programa de educación emocional que se ha diseñado específicamente para este estudio. Consta de 4 sesiones de 50 minutos, aplicadas semanalmente en la hora de tutoría. Todas las sesiones han sido aplicadas por la misma persona y en el mismo centro educ aprendizaje cooperativo y actividades grupales.

Método de intervención en educación emocional que se implementa en la escuela durante 6

sesiones. Se ha empleado una adaptación de una guia diseñada por las autoras en la que se plantean tareas para mejorar las competencias emocional Las actividades estan divididas en 5 bloques: Expresión de emociones, regulación emociona habilidades socioemocionales y autonomía emocional.

Programa de Inteligencia emocional que se estructura en relacion con el marco teórico de IER. Las actividades se han llevado a cabo en 8 sesiones en el horario lectivo y se han incluido diferentes técnicas: dramatizaciones, reflexión individual, dinámica de grupos, reflexión grupal, etc.;
Promoción del bienestar subjetivo a largo plazo, concretamente, la satisfacción con la

Beneficios a corto plazo para el incremento de la autoestima y de la conciencia ocional.

En la evaluación intragrupo, mejora de la utoestima y conciencia emocional. En concreto, mayor puntuación en habilidades sociales y mayor capacidad para no esconder emociones, mayor conciencia corporal y autoestima positiva. Mejoras en la autoestima y en habilidades sociales.

Mejora de la competencia emocional de los alumnos: empatía, respeto hacia los demás, técnicas de autocontrol, normas básicas de comportamiento, técnicas de relajación, etc.

Mejoran los problemas de conducta persistentes.

Incremento significativo en la calificación de Incremento significativo en habilidades adaptativas.

Descenso en el registro de síntomas comportamentales, aumento en el registro académico global e incremento en afecto positivo. 


\begin{tabular}{|c|c|}
\hline $\begin{array}{l}\text { FILELLA, CABELLO, } \\
\text { PÉREZ-ESCODA Y } \\
\text { ROS-MORENTE } \\
(2016)\end{array}$ & $\begin{array}{l}\text { Evaluar los efectos del videojuego } \\
\text { Happy 8-12 sobre las competencias } \\
\text { emocionales, los niveles de ansiedad, } \\
\text { el clima, y el rendimiento de los } \\
\text { estudiantes, así como la valoración de } \\
\text { todo procedimiento de los profesores } \\
\text { y los alumnos. }\end{array}$ \\
\hline $\begin{array}{l}\text { GARAIGORDOBIL Y } \\
\text { PEÑA-SARRIONANDIA } \\
(2015)\end{array}$ & $\begin{array}{l}\text { Diseñar un programa de inteligencia } \\
\text { emocional para adolescentes y } \\
\text { evaluar sus consecuencias sobre } \\
\text { variables que se relacionan con la } \\
\text { prevención de la violencia. }\end{array}$ \\
\hline $\begin{array}{l}\text { ECHEVERRÍA, LÓPEZ- } \\
\text { LARROSA Y MENDIRI } \\
(2015)\end{array}$ & $\begin{array}{l}\text { Reconocer cambios en las fortalezas } \\
\text { y problemas conductuales y } \\
\text { emocionales de los estudiantes } \\
\text { posteriormente a la aplicación del } \\
\text { programa. }\end{array}$ \\
\hline
\end{tabular}

\section{4 del alumnado participante (RAG). \\ Diseño cuasi-experimental con pre-test \\ chicos/273 y post-test y con un grupo de control. \\ Edad/curso $=5^{\circ}$ y $\quad$-Cuestionario de Desarrollo Emociona de primaria, (CDE_9-13). \\ 10-11 años. - Stait-Trait Anxiety Inventory for Grupo de control $=$ Children (STAIC). \\ Custionanio Clima sc \\ -Cuestionario el clima patio alumnado. \\ -Registro anecdótico de conductas \\ conflictivas en el patio. \\ -Calificaciones (notas) de las \\ asignaturas instrumentales. \\ -Cuestionario de valoración Happy 8-12 \\ del alumnado. \\ -Cuestionario de valoración Happy 8-12}

del profesorado.

$\mathrm{n}=148 \quad$ Estudio de diseño cuasi-experimental hicos/ 8

chicas)

Edad $=13-16$

años

$\mathrm{GC}=65$
$\mathrm{GE}=83$

\section{$n=24$}

Edad/ curso

educativo $=10^{\circ}$
Primaria, $5-7$

años. con medidas repetidas pretest-postest y grupos de control.

Instrumentos de medida:

-Trait Meta-Mood Scale (TMMS-24).

-Cuestionario de Estrategias Cognitivas

de Resolución de Situaciones Sociales

(EIS).

-State-Trait Anger Expression Inventory in Children and Adolescents (STAXI-

NA).

-Cuestionario de evaluación de la

capacidad de análisis de sentimientos (CECAS).

Diseño cuasi-experimental de grupo de control no equivalente.

Instrumentos de medida:

-Cuestionario "The Strengths and Difficulties" SDQ- self.
El software Happy 8-12, se trata de un videojuego que ha sido diseñado para que los niños aprendan a gestionar las competencias emocionales y de este modo, puedan responder de manera asertiva a los conflictos que puedan surgir en su vida. Se presentan 25 conflictos, 15 se desarrollan en el contexto escolar y 10 entre hermanos en el contexto familiar.

Programa para desarrollar la Inteligencia Emocional en adolescentes. Ha consistido en sesiones de 20 horas de duración y está compuesto de 31 actividades que se distribuyen en 5 bloques diferenciados: autoconciencia, regulación de las emociones, estado de ánimo, comunicación y empatía.

Programa socioemocional CODIP-R, aplicado en el contexto escolar. Contiene dos bloques temáticos: el primero, se centra en identificar, expresar regular las emociones, y el modo en que comprendemos las de los demás; el segundo, se
Mejora de las competencias emocionales de manera significativamente.

Descenso significativo en el nivel de

ansiedad-estado.

Mejora del clima social y el rendimiento escolar.

Mejora significativa del rendimiento

en Lengua Castellana.

Aumento del interés y de la satisfacción después de seguir con el entrenamiento del programa.

Sexo y curso, tuvieron un efecto directo en las puntuaciones finales a favor de las niñas $y$ de los alumnos de $6^{\circ}$.

El número de conflictos en el patio

disminuye un $16 \%$ y, por tanto, mejora el

clima de patio.

Incide de manera positiva en los niveles de ansiedad en el aula, mejorando el clima de aula, el rendimiento académico y la resolución asertiva de conflictos.

Aumento de la IE (atención emocional, claridad y reparación).

Aumento de las tácticas asertivas de resolución de conflictos sociales y reducción de tácticas agresivas.

Mejora positivamente el control de la ira, pero no ha sido estadísticamente significativo.

Mejora de la capacidad para analizar las causas y consecuencias de las emociones negativas. Mejora la competencia para identificar maneras constructivas de afrontarlas.

Cambio semejante originado en ambos géneros, a excepción de la atención la geridad emocional y las habilidades de interacción asertiva, que los niños mejoran. Mejora del estrés.

Los padres consideraron positivo y primordial aproximarse a los contenidos que se relacionan con las emociones y la resolución práctica de conflictos. 

$(2014$

MERCHÁN, BERMEJO Y DE DIOS (2014)

FILELLA-GUIU AGULLO, PEREZ ESCODA Y ORIOL (2014)
Lugar= Un centro

- Cuestionario de seguimiento.

Desarrollar las primeras habilidades sociales.

Demostrar los efectos positivos de la puesta en práctica de un programa puesta en práclica de un prog emocional.

Analizar el impacto de un programa de Educación Emocional en el desarrollo de las competencias emocionales, teniendo en cuenta el sexo del alumnado. educativo de la

Ciudad de A

$\mathrm{GC}=11$

$\mathrm{GE}=13$

-Grupo de discusión formado por

madres y padres y la orient

Edad/curso

escolar $=6^{\circ}$

Educación

Primaria, 10-11

años.

Lugar= I. E. María

de Lourdes del

distrito de

Pomalca

(provincia de
Chiclano).

$n=78$ (42 chicas/ Enfoque cualitativo con un grupo de

36 chicos)

Edad $=5-7$ años

\section{$\mathrm{n}=423$}

Edad $=6-12$ años

Lugar $=8$ centros

de Educación

Primaria de

Barcelona y

leida.

Grupo de control= control y uno experimental.

Instrumentos de medida:

-Test sociométrico de amistad.

-Escala de Inteligencia Emocional de Abouin (2009). prueba y post-prueba para medir las Instrumentos de medida:

-Test (Prueba pre y post).

Diseño cuasi-experimental con dos grupos pretest-postest, con grupo de control.

Instrumentos de medida:

-Escala de observación ad hoc del desarrollo emocional.

-Inventario de inteligencia emocional BarOn-ICE:NA

-Valoración del estudiantado de manera oral y mediante una escala likert.
Diseño pre-experimental con pre- centra en las distintas maneras de resolver los conflictos.

Cada sesión ha tenido una duración de 50 minutos y su periodicidad ha sido semanal.

Programa "Inteligencia Emocional". Agrupa actividades para trabajar diferentes cuestiones: autoestima, comunicación interna positiva, con emocional, conversación, sentido del humor, comunicación no verbal y los buenos modales. Se han llevado a cabo en 12 sesiones. Además, integra 9 habilidades relacionadas con lo anteriormente mencionado que se han desarrollado en las sesiones, hasta que las alumnas lograban incorporar las conductas.

Programa de Inteligencia Emocional, diseñado para la intervención.

Las actividades se centran en asuntos próximos y atractivos para el estudiantado, y exponen

situaciones que se viven en el centro escolar, con familia y amigos. Se estructuran seis bloques de contenido: Toma de contacto y expresión e identificación de emociones propias y en los demás Autoestima; Emociones positivas y negativas; Empatía; Comunicación; Resolución de conflictos. Se desarrollan en 11 sesiones de 50 minutos.

Programa de educación emocional organizado en relación con el marco teórico del GROP. Consider cinco bloques de contenidos: conciencia emocional, regulación emocional, autonomía emocional, competencia social y competencias de vida bienestar.

Se han llevado a cabo 20 actividades, dentro de la acción tutorial, de diversos tipos: análisis de de las acción tutorial, de diversos tipos: análisis de casos, pequeñ en 4 para cada bloque.
Se confirma que tratar los contenidos socioemocionales y entrenar las

competencias emocionales, en la escuela, es importante para el óptimo desarrollo individual y social de los estudiantes.

Gran progreso en relación con el desarrollo de las habilidades sociales. En este sentido, se considera que la buena autoestima, la comunicación interna positiva, el control de las emociones y los buenos modales han influido positivamente en la escucha, la conversa y la enunciación de cuestiones. Además, se aprecia una mejora en la relación estudiantes-docentes.

Se confirma que la educación emociona favorece la adquisición de competencias avocion socias de amistad la cose Mejí

sociales de amistad la clase. Mejoría en la participacion y el respeto entre los alumnos yeldocente, por lo que mejora la

convivencia del aula.

Se confirma la relación entre la inteligencia emocional y la aceptación social.

Mejora del autocontrol, la empatía y habilidades sociales.

En general, mejora de los conocimientos y competencias emocionales.

6- 8 años: Aumento relevante en todas las competencias emocionales trabajadas:

conciencia emocional, regulación emocional, autonomía emocional,

competencia social y competencias de vida y bienestar.

8-12 años: Mejoran los factores intrapersonales, interpersonales, en adaptabilidad, impresión positiva e inteligencia emocional.

La gestión del estrés no muestra un avance significativo. 


\begin{tabular}{|c|c|c|c|}
\hline $\begin{array}{l}\text { RUIZ-ARANDA, } \\
\text { MARTÍN, CABELLO, } \\
\text { PALOMERA Y } \\
\text { FERNÁNDEZ- } \\
\text { BERROCAL (2012) }\end{array}$ & $\begin{array}{l}\text { Analizar las consecuencias de un } \\
\text { programa educativo de Inteligencia } \\
\text { Emocional sobre la adaptación } \\
\text { psicosocial de los adolescentes. }\end{array}$ & $\begin{array}{l}\mathrm{n}=147(41 \\
\text { chicas/ } 28 \\
\text { chicos) } \\
\text { Edad/curso= } \\
\text { ESO, } 14-15 \\
\text { años } \\
\text { GC }=69 \\
\text { GE }=78\end{array}$ & $\begin{array}{l}\text { Diseño cuasi-experimental. } \\
\text { Instrumentos de medida: } \\
\text {-Behavior Assessment System for } \\
\text { Children and Adolescents (BASC). }\end{array}$ \\
\hline $\begin{array}{l}\text { DE DIOS Y MERCHÁN } \\
\text { (2011) }\end{array}$ & $\begin{array}{l}\text { Desarrollar, poner en práctica, } \\
\text { analizar y comparar los resultados del } \\
\text { Programa de Inteligencia Emocional, } \\
\text { analizando el impacto en el desarrollo } \\
\text { de la Competencia Lingüística y } \\
\text { Matemática, y de otros factores que } \\
\text { favorezcan el proceso de enseñanza- } \\
\text { aprendizaje. }\end{array}$ & $\begin{array}{l}n=95 \\
\text { Edad/curso } \\
\text { escolar }=4^{\circ} \\
\text { Educación } \\
\text { Primaria, 8-9 } \\
\text { años. } \\
\text { Lugar= dos } \\
\text { colegios públicos } \\
\text { de Badajoz. }\end{array}$ & $\begin{array}{l}\text { Estudio transfronterizo con grupo } \\
\text { experimental y grupo de control. } \\
\text { Instrumentos de medida: } \\
\text {-Sociograma (Test de amistad). } \\
\text { - Test de Habilidades de Inteligencia } \\
\text { Emocional (Autoinforme) } \\
\text {-Ficha de evaluación. }\end{array}$ \\
\hline $\begin{array}{l}\text { AGULLÓ, FILELLA, } \\
\text { SOLDEVILA Y RIBES } \\
\text { (2011) }\end{array}$ & $\begin{array}{l}\text { Evaluar un Programa de Educación } \\
\text { Emocional para ayudar a prevenir los } \\
\text { efectos de las emociones negativas y } \\
\text { facilitar la relación con él mismo y con } \\
\text { los demás. }\end{array}$ & $\begin{array}{l}\mathrm{n}=510 \\
\text { Edad= } 8-10 \text { años } \\
\text { Lugar= centros } \\
\text { educativos de } \mathrm{E} \text {. } \\
\text { Infantil y } \mathrm{E} . \\
\text { Primaria de la } \\
\text { provincia de } \\
\text { Lleida. }\end{array}$ & $\begin{array}{l}\text { Metodología cuasi-experimental de dos } \\
\text { grupos con pretest y postest. } \\
\text { Instrumentos de medida: } \\
\text {-El cuestionario (CEEMP) } \\
\text {-La Entrevista. } \\
\text {-Ficha de observación. } \\
\text {-Cuestionario valorativo de cada } \\
\text { actividad. }\end{array}$ \\
\hline
\end{tabular}

Programa de entrenamiento de Inteligencia Emocional denominado "Proyecto INTEMO" se basa en el modelo de habilidades de Mayer Salovey (1997). Se ha llevado a cabo una metodologia lúdica, atractiva, vivencial, práctica, grupal, dinamica y participativa.

La intervención ha consistido en una sesión de 1 hora a la semana durante 10 semanas (durante dos cursos académicos). Se ha realizado como parte del horario regular de las clases.

Programa "Desarrollando la Inteligencia Emocional" (DIE), elaborado para ser implementado dentro de aula e integrado al currículo del alumnado. Está dividido en 6 bloques: ¿Cómo te sientes?; conseguir autoconciencia emocional; ¿cómo se sienten los demás? aprendo las habilidades en demás y mejoro racios demás y mjo relacion 1 inión por seman. Se han levado a cabo durante 1 sesión por semana. Se trata de tareas cercanas a la vida del niño y la actividades escrilas se complementan con dinámicas de grupo y tareas de ejecución. Programa de Educación Emocional (PEEP). Los contenidos coinciden con los cinco bloques de la educación emocional y se proponen cinco actividades de cada bloque que se llevan a cabo en las horas escolares (durante una hora semanal en el curso escolar). Se propone el desarrollo de estrategias y habilidades con el objetivo de participar en el desarrollo de la educación emocional.
Alto nivel de agrado de los profesores y alumnos.

Las niñas de 6-8 años obtienen una puntuación más elevada que los niños en: Regulación emocional, Competencia social y Competencia de vida y bienestar. Resultado positivo sobre las variables: ansiedad, estrés social, depresión, somatización y autoestima.

Ningún efecto sobre las variables de autoconfianza y vínculos con los padres.

Confirma la relación entre inteligencia

emocional, rendimiento escolar y resolución de conflictos.

Reducido avance en los índices de amistad y aceptación de los alumnos con NEE, sobre todo en aquellos cuyas condiciones sociales y personales son desfavorables (inmigrantes).

La totalidad de bloques temáticos analizados presentan una mejora positiva. La consciencia y la regulación emocional son las areas que más se han visto favorecidas.

Aumenta el aprendizaje de conocimientos emocionales y se considera que su puesta en práctica, con el tiempo, mejoraría las competencias emocionales.

La participación y actitud mejora, as como también la relación consigo mismo y con los demás. 


\section{Discusión}

A continuación, se analiza y compara la información destacable extraída de los documentos seleccionados para llevar a cabo la revisión bibliográfica con el objetivo de extraer relaciones y conclusiones. Para ello, se ha dividido la información en tres variables: variables de identificación (autoría y año de publicación), variables metodológicas (objetivos, muestra grupo de control, metodología, instrumentos, contenidos y duración) y variables de contenido (resultados).

Atendiendo a las variables de identificación, se destaca que la totalidad de las intervenciones, exceptuando la de Pastor-Arnau (2019) y Gálvez-Iñiguez (2018), se han realizado en colaboración con otros autores. Además, se pueden identificar algunos autores de referencia en el ámbito de la educación emocional, pues han publicado diversos documentos que se centran en esta temática como, por ejemplo, Filella con 32 trabajos, Pérez-Escoda con 29 artículos y Cejudo con 16 publicaciones. Asímismo, cabe indicar que el número de mujeres (82\%) es bastante superior al de los hombres (18\%), con lo cual, desde una perspectiva de género, es positivo.

Con respecto al año de publicación, se observa un equilibrio en el número de intervenciones relacionadas con el tema analizado, sin embargo, se aprecia un leve incremento en los últimos años. En el intervalo 2011-2012, la curva de publicaciones se mantiene estable, entre cero y dos publicaciones por año, excepto en 2014, que se han publicado tres artículos (Agulló et al., 2011; De Dios y Merchán, 2011; Echeverría et al., 2015; Filella-Guiu et al., 2014; Filella et al., 2016; Garaigordobil y Peña-Sarrionandia, 2015; Merchán et al., 2014; Ruiz-Aranda et. al., 2012; Torres y Piscoya, 2014). Además, desde el 2017 hasta la actualidad (2021), el pocentaje de publicaciones (57'14\%) es elevado con respecto a los anteriores, pero no es significativo (Aguilar et al., 2021; Cabello-Cuenca et al., 2019; Echeverría et al., 2020; Gálvez-Iñiguez, 2018; García y Andrés-Roqueta, 2017; Martínez-Álvarez et al., 2020; Pastor-Arnau, 2019; PostigoZegarra et al., 2019; Sigüeña-Marín et al., 2019; Tur-Porcar et al., 2021). Es en el año 2019 cuando se observe un leve incremento en la publicación de artículos $(n=4)$ (Cabello-Cuenca et al., 2019; Pastor-Arnau, 2019; Postigo-Zegarra et al., 2019; Sigüeña-Marín et al., 2019).

En relación con las variables metodológicas, se indica, en primer lugar, que todos los estudios coinciden en establecer objetivos semejantes pues, en general, tratan de analizar el impacto de un programa de educación emocional, socioemocional o de inteligencia emocional que fomenta el desarrollo de las competencias emocionales. No obstante, unos se dirigen a valorar el ajuste psicosocial (Cejudo, 2017; Ruiz-Aranda, 2012), el desarrollo de habilidades sociales (Gálvez-Iñiguez, 2018; Torres y Piscoya, 2014) y la mejora de los problemas conductuales y de la convivencia en el aula (Aguilar, et al., 2021; Agulló et al., 2011; Cabello-Cuenca et al., 2019; Echeverría et al., 2020; Filella et al., 2016; Garaigordobil y Peña-Sarrionandia, 2015; García y Andrés- Roqueta, 2017; Postigo-Zegarra et al., 2019; Schoeps et al., 2018; Tur-Porcar et al., 2021). Otros, una minoría, además, analiza la efectividad sobre el rendimiento académico y la salud mental (Sigüeña-Marín et al., 2019), empatía (Garaigordobil y Peña-Sarrionandia, 2015; Sigüeña-Marín et al., 2019) y autoestima (Aguilar, et al., 2021; Gálvez-Iñiguez, 2018; Sigüeña-Marín et al., 2019; Torres y Piscoya, 2014).

Así, en lo referido al número de participantes, hay casos donde las diferencias entre los estudios analizados llegan a ser considerables. Un $28{ }^{\prime} 57 \%$ se sitúa por debajo de los 95 participantes. En primer lugar, en el artículo de García y Andrés-Roqueta (2017) y Echeverría et al. (2015) hay un total de 24 individuos. Le sigue Aguilar (2021), con una 
intervención de 58 miembros y Merchán et al. (2014) con 78. Por último, Echeverría et al. (2020) cuenta con 83 en total, Pastor-Arnau (2019) con 88 y De Dios y Merchán (2011) con 95 participantes.

Por otra parte, destacan las publicaciones cuya muestra es superior a 100 individuos. Por un lado, se ha de prestar atención al estudio de Sigüeña-Marín et al. (2019) con 104 sujetos y al de Cejudo (2017) y Gálvez-Iñiguez (2018), con una muestra de 123. De la misma manera, Torres et al. (2014) cuenta con 145 sujetos y Ruiz-Aranda (2012) con 147. Además, hay dos estudios (Garaigordobil y Peña-Sarrionandia, 2015; Schoeps et al., 2018) que disponen de 148 participantes. Asimismo, Martínez-Álvarez et al. (2020) dispone de una muestra de 198 y Postigo-Zegarra et al. (2019) de 293. Les sigue el artículo de Cabello-Cuenca et al. (2019) con 301 miembros y el de Filella-Guiu et al. (2014) con 423. Por último, hay tres artículos con más de 500 participantes, por un lado, el de Agulló et al. (2011) cuenta con 510, el de Tur-Porcar et al. (2021) con 555 sujetos y el de Cabello-Cuenca et al. (2019) con 1477 miembros.

Cabe mencionar el artículo de Cabello-Cuenca et al. (2019), ya que se trata de pues se trata de una intervención doble en la que participan 574 alumnos de Educación Primaria y 903 alumnos que cursan Educación Secundaria Obligatoria. Es decir, el estudio se lleva a cabo de manera independiente a través de la puesta en práctica de dos programas adaptados a la edad de los participantes.

Además, es necesario destacar que se aprecian algunos artículos que disponen de un grupo de control (Aguilar, 2021; Agulló et al., 2011; Cabello-Cuenca et al., 2019; Cejudo, 2017; De Dios y Merchán, 2011; Echeverría et al., 2015; Filella-Guiu et al., 2014; GálvezIñiguez, 2018; Garaigordobil y Peña-Sarrionandia, 2015; Merchán et al., 2014; PastorArnau, 2019; Ruiz-Aranda, 2012; Schoeps et al., 2018; Sigüeña-Marín et al., 2019; TurPorcar et al., 2021), y otros que no lo incluyen (23'81\%) (Echeverría et al., 2020; García y Andrés-Roqueta 2017; Martínez-Álvarez et al., 2020; Postigo-Zegarra et al., 2019; Torres et al., 2014).

Por lo que se refiere al género de los participantes, se destaca que en más de la mitad de los artículos (57'14\%) no se estudia esta variable y, por consiguiente, no se especifica. Por contra, en 11 artículos (Andrés-Roqueta, 2017; Cabello-Cuenca et al., 2019; Filella-Guiu et al., 2014; Filella et al., 2016; Gálvez-Iñiguez, 2018; Garaigordobil y Peña-Sarrionandia, 2015; García y Martínez-Álvarez et al., 2020; Merchán et al., 2014; Postigo Zegarra et al., 2019; Ruiz-Aranda et al., 2012; Schoeps et al., 2018) se diferencian los participantes según su género y, aunque es mayor la intervención de los sujetos de género femenino, no hay mucha diferencia con respecto a los de género masculino. Es decir, se puede afirmar que, en lo que respecta al género de los participantes, la tendencia es igualitaria y equilibrada.

Con referencia a la edad de la muestra, los artículos se clasifican según la etapa escolar de los participantes. En general, tienen una edad comprendida entre los 5 hasta los 16 años, pues la muestra utilizada se corresponde con alumnos que cursan las etapas escolares obligatorias, es decir, Educación Primaria (10- 6a de Educación Primaria), el $66{ }^{\prime} 67 \%(n=14)$ y Educación Secundaria Obligatoria, el $28 ' 57 \%(n=6)$. Sin embargo, en la intervención de Cabello-Cuenca et al. (2019) participa tanto alumnado de Educación Primaria, como alumnado de Educación Secundaria Obligatoria.

Por un lado, en cuanto a los escolares en la etapa de primaria, se encuentran los que pertenecen a 1ㅇ, con edades incluidas entre los 6 y 7 en Echeverría et al. (2020) y entre los 6 y 8 en García y Andrés-Roqueta (2017). Seguidamente, la intervención de Merchán 
et al. (2014) y Echeverría et al. (2015) con individuos de $1^{\circ}$ y $2^{\circ}$, que abarcan una edad de 5 a 7 años. A continuación, en Gálvez-Iñiguez (2018) se incluyen aquellos que pertenecen a 3ํㅡ y $4^{\circ}$ y tienen entre 8 y 9 años, como en De Dios y Merchán (2011), aunque en su muestra participan únicamente sujetos de $4^{\circ}$ de primaria. En el trabajo de Agulló et al. (2011), los participantes pertenecen a los niveles de entre 3ํy y $5^{\circ}$ y su edad es de 8 hasta 10 años. Se puede apreciar que en Torres y Piscoya (2014) y Filella (2016), se recurre a una muestra de 10 y 11 años, por lo cual cursan $5^{\circ}$ y $6^{\circ}$, y en Cejudo (2017) y Aguilar et al. (2021), los sujetos pertenecen a 6으 y comprenden edades entre los 10 y los 12 años. En último lugar, se ha de mencionar el documento de Filella-Guiu et al. (2014) y Martínez-Álvarez et al. (2020), con sujetos de 6 hasta 12 años, que abarcan el conjunto de las etapas educativas de Primaria (1-은 y el artículo de TurPorcar et al. (2021), cuya población tiene de 7 a 12 años y cursa desde $2^{\circ}$ a $6^{\circ}$ de primaria.

Por otro lado, en lo que respecta a la etapa de Educación Secundaria Obligatoria (ESO), resaltan las intervenciones de Postigo-Zegarra et al. (2019) y Schoeps et al. (2018), con participantes de 12 a 15 años que cursan $1^{\circ}$ y $2^{\circ}$ de ESO. Posteriormente, el de PastorArnau (2019) y el de Sigüeña-Marín et al. (2019), con una muestra de 13 y 14 años escolarizados en $2^{\circ}$ y $3^{\circ}$ de ESO. En el artículo de Garaigordobil y Peña Sarrionandia (2015), se implican sujetos de 13 a 16 años que cursan $2^{\circ}$ y $4^{\circ}$ ESO y, para finalizar, el trabajo de Ruiz-Aranda et al. (2012) incluye una muestra de sujetos de 14 y 15 años que pertenecen a $3^{\circ}$ y $4^{\circ}$ ESO. Se ha de destacar la intervención de Cabello-Cuenca (2019), ya que comprende tanto participantes de la etapa de educación primaria, de $5^{\circ}$ y $6^{\circ}$ (1011 años), como de la etapa de educación secundaria con una muestra de $1^{\circ}$ y $2^{\circ}$ ESO (12-13 años).

Con referencia a la metodología de las intervenciones, se observa una preferencia por los estudios cuasi-experimentales (71'43\%). Se encuentran en Agulló et al. (2011), RuizAranda et al. (2012), Filella-Guiu et al. (2014), Echeverría et al. (2015), Garaigordobil y Peña- Sarrionandia (2015), Filella et al. (2016), Cejudo (2017), García y Andrés-Roqueta (2017), Gávez-Íñiguez, Schoeps et al. (2018), Cabello-Cuenca et al. (2019), PastorArnau (2019), Sigüeña-Marín et al. (2019), Echeverría et al. (2020) y Tur-Porcar et al. (2021). Además, llama la atención el estudio de Aguilar et al. (2021), pues lleva a cabo un estudio longitudinal "ex post facto" cuasi-experimental.

Al mismo tiempo, diversos trabajos (23'8\%) se centran en la realización de investigaciones de una tipología variada: un estudio transfronterizo (De Dios y merchán, 2011); una investigación con un enfoque cualitativo (Merchán et al., 2014); un diseño pre-experimental (Torres y Piscoya, 2014); una investigación de diseño mixto que integra datos cualitativos y cuantitativos (Postigo-Zegarra et al., 2019) e incluso un estudio descriptivo, comparativo y correlacional (Martínez-Álvarez et al., 2020).

En lo que concierne a los instrumentos de medida empleados, se puede afirmar que se utilizan una gran variedad, tanto para el comienzo como para el final de las intervenciones, pues todos ellos hacen uso de cuestionarios o escalas de valoración para evaluar el desarrollo emocional de los escolares o el progreso de habilidades sociales (valoración del comportamiento social). En este sentido, se aprecian estos instrumentos: CEEMP (Agulló et al. 2011), Test sociométrico de amistad (Aguilar et al., 2021; De Dios y Merchán, 2011; Merchán et al., 2014) y Test de Habilidades de Inteligencia emocional (De Dios y Merchán, 2011), BASC (Cejudo, 2017; Ruiz-Aranda et al., 2012), Escala de observación del desarrollo emocional y ICE:NA (Filella-Guiu et al. 2014), Escala de Inteligencia Emocional de Abouin (Merchán et al., 2014), Cuestionario "The Strengths and Difficulties" (Echeverría et al., 2015), TMMS-24 
(Garaigordobil y Peña-Sarrionandia, 2015; Postigo-Zegarra et al., 2019; Sigüeña-Marín et al., 2019).

Además, con la misma finalidad, se hacen uso de se hace uso con la misma finalidad de los siguientes: EIS, STAXI-NA y CECAS (Garaigordobil y Peña-Sarrionandia, 2015); CDE_9-13 (Filella et al., 2016; Pastor-Arnau, 2019), STAIC, Cuestionario Clima Social de Aula y de patio (Filella et al., 2016), TEIQue-ASF (Cejudo, 2017), EMOCIONALTEST (García y Andrés- Roqueta, 2017), MESSY y escala autoestima de Rosenberg (Gálvezİñiguez, 2018; Sigüeña-Marín, et al. 2019), PANASN (Cejudo, 2017), EAQ-30 y FAS (Gálvez-Íñiguez, 2018), ESCQ, CYB-VIC y CYB-AG (Schoeps et al., 2018), CDE y STAI (Cabello-Cuenca et al. 2019), Escala de autoconcepto de Piers-Harris, SPANE (PostigoZegarra et al., 2019), SDQ (Sigüeña-Marín et al., 2019), CCE (Echeverría et al., 2020), BAS 1 (Martínez-Álvarez et al., 2020), Cuestionario de relaciones socioafectivas.(Aguilar et al., 2021); Escala de Agresividad Física y Verbal, Escala de Inestabilidad Emocional, Mann-Whitney U Test. y WSRT (Tur-Porcar et al., 2021). Asimismo, algunas intervenciones (19\%), consideran las calificaciones escolares, de manera que una de ellas (Cejudo, 2017) emplea el RAG y las demás únicamente comparan las calificaciones anteriores y posteriores a la investigación (Cabello-Cuenca et al., 2019; Filella et al., 2016; Pastor-Arnau, 2019).

Con respecto a los contenidos trabajados en la intervención y a las dinámicas implementadas, todos ellos se apoyan en una metodología lúdica, activa, vivencial, práctica, grupal y participativa cuya finalidad es descubrir y profundizar en el ámbito de las emociones. Sin embargo, se ha de prestar atención los contenidos escogidos en cada uno de los Programas de Educación Emocional. En este sentido, solamente en el artículo de Echeverría et al. (2020) se hace uso de una mascota en sus sesiones y en un $33^{\prime} 33 \%$ de los artículos han preferodo llevar a cabo tareas considerando los cinco bloques temáticos relacionados con las competencias emocionales que siguen en en GROP desde 1997: conciencia emocional, regulación emocional, autonomía emocional, competencia social y competencias de vida y bienestar (Agulló et al., 2011; Filella-Guiu et al., 2012; Garaigordobil y Peña-Sarrionandia, 2015; García y Andrés-Roqueta, 2017; Martínez-Álvarez et al., 2020; Pastor-Arnau 2019).

Siguiendo con lo anterior, en tres intervenciones (Ruiz-Aranda et al., 2012; PostigoZegarra et al., 2019; Sigüeña-Marín et al., 2019) siguen el modelo de Mayer y Salovey (1997) y, por ello, dividen las actividades en cuatro bloques temáticos vinculados con las cuatro competencias emocionales (percepción emocional, facilitación emocional del pensamiento, comprensión y a análisis de las emociones y regulación emocional) que establecen dichos autores. Por último, un 57'14\% ( $n=12)$, emplea contenidos diversos relacionado con el manejo emocional, la empatía, la autoestima. Además, en CabelloCuenca et al. (2019), Echeverría et al. (2015), Merchán et al. (2014), Torres y Piscoya (2014), Tur-Porcar et al. (2021) se trabaja la comunicación y la resolución asertiva de y se han de tener en cuenta aquellas intervenciones específicas que trabajan las emociones a través de la música (Pastor-Arnau, 2019) y la expresión corporal (Aguilar et al., 2021).

En relación con la duración y el momento de las intervenciones, es necesario indicar que todas ellas se llevan a cabo en un centro escolar. Además, en algunos estudios indican que la intervención se ha desarrollado en las horas escolares regulares (acción tutorial) y comprenden los contenidos del currículo académico (Agulló et al., 2011; Cejudo, 2017; De Dios y Merchán, 2011; Filella-Guiu et al., 2014; Ruiz-Aranda et al., 2012). Así, todas tienen una duración inferior o igual a la de un curso académico, a excepción de la intervención de Ruiz-Aranda et al. (2012) que su duración es de dos cursos escolares (10 semanas). 
Comenzando con Gálvez-Íñiguez (2018) que realiza la intervención más corta, 4 sesiones; seguidamente, se encuentran aquellas que con una duración de 6 sesiones (Echeverría et al., 2020; García y Andrés-Roqueta, 2017), 8 sesiones (Cejudo, 2017; Sigüeña-Marín et al., 2019), 11 sesiones (Merchán et al., 2014; Postigo-Zegarra et al., 2019; Schoeps et al., 2018), 12 sesiones (Aguilar et al., 2021; Torres y Piscoya, 2014), 16 sesiones (Tur-Porcar et al., 2021) y 20 sesiones (Garaigordobil y Peña-Sarrionandia, 2015). Además, se aprecia que algunas investigaciones se desarrollan durante 1 sesión semanal (De Dios y Merchán, 2011; Echeverría et al., 2015), pero no se especifica el número total de sesiones u horas empleadas. Por último, se encuentran aquellos trabajos que abarcan un cuatrimestre (Pastor-Arnau, 2019) y un curso académico completo (Agulló et al., 2011; Martínez- Álvarez et al., 2020). Para finalizar, se ha de prestar atención a aquellos que su duración coincide con la realización de las actividades: 20 actividades (Filella-Guiu et al., 2014) y 25 conflictos/actividades (Cabello-Cuenca, 2019; Filella et al., 2016).

Por último, se especifican las variables de contenido, es decir, los resultados obtenidos. En primer lugar, un $47^{\prime} 62 \%$ de los estudios demuestran que, tras aplicar los programas de educación emocional, se interiorizan conocimientos emocionales, y aumentan y mejoran las competencias emocionales (conciencia emocional, regulación emocional, autonomía emocional y competencias de vida y bienestar) de los niños y niñas (Agulló et al., 2011; Cabello-Cuenca et al., 2019; Cejudo, 2017; Filella et al., 2016; GálvezÍñiguez, 2018; García y Andrés-Roqueta, 2017; Martínez-Álvarez et al., 2020; Merchán et al., 2014; Postigo-Zegarra et al., 2019; Schoeps et al., 2018). Sin embargo, aunque en Agulló et al. (2011), se indicar el aprendizaje de conocimientos emocionales, se establece la necesidad de un periodo mayor para verificar la mejora de dichas competencias; además, en Schoeps et al. (2018) el programa no ha sido efectivo en el desarrollo de competencias emocionales, sin embargo, mejora la capacidad para percibir, comprender y regular las emociones. En esta misma línea, los efectos de algunos artículos (Cejudo, 2017; Garaigordobil y Peña-Sarrionandia, 2015) indican que la intervención ha incrementado notablemente la Inteligencia Emocional de los escolares. Así pues, esta mejora puede suponer un aumento significativo en la competencia social (Filella-Guiu et al., 2014), una mejora del autocontrol, la empatía, respeto a los demás y, en general, de las habilidades sociales (Merchán et al., 2014; Torres y Piscoya, 2014) y habilidades adaptativas (Cejudo, 2017).

De igual modo, estos resultados coinciden con estudios previos, pues Rubiales et al. (2018) en su revisión de la literatura establece que, respecto a la competencia emocional, seis de los diez estudios evidenciaron efectividad en los programas. También lo confirman Catalano et al. (2002) Wilson \& Lipsey (2007) Weissberg et al. (2007) en sus investigaciones.

Asimismo, en el 42 ' $86 \%$ de las publicaciones se consiguen resultados positivos sobre las variables de ansiedad, estrés social, autoestima y autoconcepto (Cabello-Cuenca et al., 2019; Echeverría et al., 2015; Filella-Guiu et al., 2014; Filella et al., 2016; GálvezÍñiguez, 2018; Pastor-Arnau, 2019; Ruiz-Aranda et al., 2012; Sigüeña-Marín et al., 2019). Sin embargo, en Aguilar et al. (2021) no se demuestra el fomento de la autoestima en los niños y niñas. Pues, según Rubiales et al. (2018), en cuatro estudios investigados, no especificados, se ha comprobado la efectividad de los programas con respecto a la salud mental, la autoestima y la autoeficacia.

Por otra parte, en un 61 ' $9 \%$ de los trabajos se mejoran las relaciones sociales, la actitud y la agresividad y, consecuentemente, aumentan las estrategias asertivas para la resolución de conflictos y se estimula la convivencia en la escuela; es decir, el clima del aula es más positivo (Aguilar et al., 2021; Agulló et al., 2011; Cejudo, 2017; Echeverría 
et al., 2020; Filella et al., 2016; Garaigordobil y Peña-Sarrionda, 2015; García y AndrésRoqueta, 2017; Martínez-Álvarez et al., 2020; Merchán et al., 2014; Pastor-Arnau, 2019; Postigo-Zegarra et al., 2019; Schoeps et al., 2018; Torres y Piscoya 2014). En general, el alumnado tiene una capacidad superior para analizar causas y consecuencias de las emociones negativas y para identificar maneras adecuadas para atender dichas emociones. En Filella et al. (2016), además, se mejora el clima social del patio, ya que los problemas descienden un 16\%. Así pues, es necesario indicar que, en Merchán et al. (2014), Torres y Piscoya (2014) y Pastor- Arnau (2019) también se mejora el vínculo entre los iguales y el profesor. En el artículo de Schoeps et al. (2018) la intervención resulta eficaz para reducir la incidencia del ciberacoso. No obstante, se ha de prestar atención a Sigüeña-Marín et al. (2019), pues no se evidenciaron mejoras en relación con los problemas comportamentales.

En esta línea, es necesario indicar que De Dios y Merchán (2011) y Merchán et al. (2014) establecen una relación entre la inteligencia emocional y la aceptación social, pues en uno de ellos (De Dios y Merchán, 2011) se encuentra un avance sutil en cuanto a los índices de amistad y aceptación de los alumnos con necesidades educativas especiales. Además, en De Dios y Merchán (2011) se observan cambios en la actitud para afrontar los conflictos familiares; sin embargo, en Merchán et al. (2014) no se observan consecuencias en la relación parental.

En investigaciones previas, los resultados fueron semejantes, puesto que mejora de manera significativa la identificación y dominio de las emociones, de tal forma que se establecen y mantienen relaciones beneficiosas. Adicionalmente, mejora la comunicación con los demás y el manejo de conflictos interpersonales (Beelmann y Losel 2006; Durlak y Wells, 1997; Durlak et al., 2008; Hahn et al., 2007; Stage y Quiroz, 1997; Wilson et al., 2001; Gansle, 2005; Wilson y Lipsey, 2007). Por lo general, mejoran las habilidades sociales y emocionales (Durlak y Wells, 1997; Wilson y Lipsey, 2007).

Finalmente, en el 23 ' $8 \%$ de los casos $(n=5)$, los resultados señalan una mejora considerable del rendimiento escolar (Cabello-Cuenca et al., 2019; Cejudo, 2017; Filella et al., 2016; Martínez-Álvarez et al., 2020; Pastor-Arnau, 2019), del mismo que en trabajos anteriores (Gil-Olarte et al., 2005, 2006).

\section{Consideraciones finales}

El objetivo principal del presente artículo era llevar a cabo una revisión bibliográfica para establecer una justificación teórica que señalara la necesidad de recurrir al área emocional para solucionar conflictos en la escuela (convivencia escolar y rendimiento académico), así como también revisar la evidencia científica que posibilitara diseñar intervenciones válidas.

Por tanto, posteriormente al análisis, se ha demostrado, en líneas generales, que la educación emocional beneficia el clima de aula disminuyendo conductas negativas), mejora el rendimiento académico y desarrolla competencias emocionales para reconocer y controlar sus emociones y mantener relaciones positivas.

Estos resultados coinciden con los obtenidos en estudios de revisión previos (Diekstra y Gravesteijn, 2008; Pena y Repetto, 2008; Rubiales et al., 2018). Así, tal y como establecen Alcoser-Grijalva, Moreno-Ronquillo y León García (2019), el desarrollo apropiado de la inteligencia emocional favorece el incremento de habilidades fundamentales para solucionar dificultades y aprender a vivir. 
Pese a los resultados positivos obtenidos, se han de indicar las limitaciones esenciales que se aprecian. En primer lugar, resalta el reducido periodo de duración de las sesiones de los programas que se implementan, pues la mayoría de ellos se desarrollan en un tiempo inferior al de un curso escolar, de manera que complica el desarrollo de la intervención y se inician a observar los resultados tras su finalización. Asimismo, a pesar de los resultados cuantitativos, se hace necesario tener en cuenta que las publicaciones ofrecen resultados a partir de la implementación de cuestionarios o tests rellenados por los propios escolares, por lo cual, no se considera la sinceridad de cada uno de ellos y las respuestas podrían estar influenciadas por aquello socialmente deseable. Se observa, de esta manera, que la puesta en práctica de las intervenciones en grandes muestras complica el proceso, por lo que se observarían mejores resultados y facilitaría el proceso requerir grupos reducidos de individuos. Del mismo modo, para que los programas de educación emocional fuesen efectivos, es fundamental la responsabilidad y la instrucción del profesorado involucrado, al igual que sucede en el trabajo de CabelloCuenca et al. (2019), Schoeps et al. (2018) y Filella-Guiu et al. (2014). En consecuencia, se precisa más tiempo para formar al profesorado en educación emocional y proveerlo de competencias emocionales. Para finalizar, se destaca la necesidad de mejorar en el estudio de la influencia que supone educar emocionalmente a los niños y niñas y adolescentes.

Es conveniente, además, mencionar el artículo de Cabello-Cuenca, Pérez-Escoda, RosMorente y Filella-Guiu (2019) por ser el más completo y reunir la mayoría de las variables analizadas que contribuyen al éxito de la intervención. Este trabajo incluye una muestra tanto de alumnado de Educación Primaria como de Educación Secundaria y, a través de un videojuego, desarrolla las diferentes competencias emocionales. Tiene en cuenta las diferentes variables analizadas: niveles de ansiedad, resolución de conflictos, clima de aula y rendimiento de los estudiantes.

En conclusión, se establece que los programas de educación emocional, socioemocional y de inteligencia emocional son beneficiosos para los niños y jóvenes en la escuela. Gracias a ello, podría mejorar de manera significativa su desarrollo emocional y social, y es decisivo para su óptimo desarrollo integral. Por todo ello, es indispensable incorporar al currículo académico contenidos para desarrollar las competencias emocionales de todos y todas.

\section{Referencias}

Aguilar, M. D., García, C. M. y Gil, C. (2021) Efectividad de un programa educativo en Educación Física para fomentar las habilidades socioafectivas y prevenir la violencia en Educación Primaria. Retos, 41, 492-501.

Agulló, M. J., Filella, G., Soldevila, A, y Ribes, R. (2011). Evaluación de la educación emocional en el ciclo medio de Educación Primaria. Revista de Educación, 354, 765-783

Baena, G. (2017). Metodología de la investigación. Grupo Editorial Patria.

Bar-On, R. (2000). Emotional and Social Intelligence: Insights from the Emotional Quotient Inventory (EQ-i). En Bar-On, R. y Parker, J. D. A. (2000). Handbook of Emotional Intelligence. Jossey-Bass.

Beelmann A. y Lösel F. (2006) Child Social Skills Training in Developmental Crime Prevention: Effects on Antisocial Behavior and Social Competence. Psicothema, 18(3), 603-10.
Bisquerra, R. (2003). Educación emocional y competencias básicas para la vida. Revista de Investigación educativa, 21(1), 7-43.

Bisquerra, R. (2006). Orientación psicopedagògica y educación emocional. Estudios sobre Educación, 11, 9-25.

Bisquerra, R. y Pérez-Escoda, N. (2007). Las competencias emocionales. Educación XXI, 10, 61-82.

Bisquerra, R. y Pérez, N. (2012). Educación emocional: estrategias para su puesta en práctica. Revista de la Asociación de Inspectores de Educación de España, 16, 1-11.

Cabello-Cuenca, E., Pérez-Escoda, N., Ros-Morente, A. y FilellaGuiu, G. (2019). Los programas de educación emocional Happy 812 y Happy 12-16. Evaluación de su impacto en las emociones y el bienestar. Revista Española de Orientación y Psicopedagogía, $30(2), 53-66$. 
Cejudo, J. (2017). Efectos de un programa de mejora de la inteligencia emocional sobre el ajuste psicosocial y el rendimiento académico en Educación Primaria. Journal for the Study of Education and Development, 40(3), 503-530

Cepa, A., Heras, D., y Fernández-Hawrylak, M. (2017). La educación emocional en la infancia: una estrategia inclusiva. Aula Abierta, 46, 73-82.

De Dios, J., y Merchán, I. M. (2011). Investigación transfronteriza sobre un programa de inteligencia emocional con alumnos de Educación Primaria de Badajoz (España) y Castelobranco (Portugal): Análisis de la eficacia del programa en alumnos de Badajoz. International Journal of Developmental and Educational Psychology, 1(1), 303-312.

Durlak, J.A., Weissberg, R.P., Dymnicki, A.B., Taylor, R.D. y Schellinger, K. (2008). The Effects of Social and Emotional Learning on the Behavior and Academic Performance of School Children.

Durlak, J. A., y Wells, A. M. (1997). Primary Prevention Mental Health Programs for Children and Adolescents: A Meta-Analytic Review. American Journal of Community Psychology, 25(2), 115152.

Echeverría, B., López, S. y Mendiri, P. (2015). Aplicación de un programa socioemocional CODIP- $\mathrm{R}$ en alumnos de primer curso de primaria. Revista de Estudios e investigación en Psicología y Educación, Extr(5), 1-4.

Echeverría, B., López-Larrosa, S., y Mendiri, P. (2020). Aplicación de un programa de educación socio-emocional para alumnado de Educación Primaria. Revista de Estudios e Investigación en Psicología y Educación, 7(2), 174-183.

Filella, G., Cabello, E., Pérez-Escoda, N. y Ros-Morente, A. (2016). Evaluación del programa de Educación Emo- cional "Happy 8-12" para la resolución asertiva de los conflictos entre iguales. Electronic Journal of Research in Educational Psychology, 14(3), 582-601.

Filella-Guiu, G., Agulló, M. J., Pérez-Escoda, N. y Oriol, X. (2014). Resultados de la aplicación de un programa de educación emocional en Educación Primaria. Estudios sobre educación, 26, 125-147. https://hdl.handle.net/10171/36786

Gansle, K. A. (2005). The Effectiveness of School-Based Anger Interventions and Programs: a Meta-Analysis. Journal of School Psychology, 43, 321-341.

Gálvez-lñiguez, Y. (2018). Eficacia de un programa de educación emocional: contaminación entre condiciones experimentales en un estudio controlado. Revista de Psicología Clínica con Niños y Adolescentes, 5(2), 17-22.

Garaigordobil, M., Peña- Sarrionandia, A. (2015). Effects of an emotional intelligence program in variables related to the prevention of violence. Frontiers in Psychology, 6(743), 1-11.

García, J. A., (2012). La educación emocional y su importancia en el proceso de aprendizaje. Revista Educación, 36(1), 97-109.

García, I., y Andrés-Roqueta, C. (2017). La educación emocional como método para mejorar los problemes de conducta de los ninos y niñas. Fòrum de Recerca, 22, 81-91.
Gil-Olarte, P., Guil, R., Mestre, J.M., y Nuñez, I. (2005). La Inteligencia emocional como variable predictora del rendimiento académico. En J. Romay y R. García (Eds.), Psicología social y problemas sociales. Psicología Ambiental, Comunitaria y Educación. Biblioteca Nueva.

Gil-Olarte, P., Palomera, R. y Brackett, M. (2006). Relating emotional intelligence to social competence and academic achievement in high school students. Psicothema, 18, 118-123.

Goleman, D. (1995). Emotional Intelligence. Why it can matter more than IQ. Bantam Books.

Hahn, R.; Fuqua-Whitley, D.; Wethington, H.; Lowy, J.; Liberman, A.; Crosby, A.; Fullilove, M.; Johnson, R.; Moscicki, E.; Price, L.; Snyder, S.R.; Tuma, F.; Cory, S.; Stone, G.; Mukhopad- haya, K.; Chattopadhyay, S. y Dahlberg, L. (2007). Effectiveness of Universal School-Based Programs to Prevent Violent and Aggressive Behavior. A Systematic Review. Am J Prev Med, 33(2), 114-129.

Martínez-Álvarez, I., Hidalgo-Fuentes, S., y Sospedra-Baeza, M. J. (2020). Análisis del nivel de adaptación socioemocional y su relación con el rendimiento en el alumnado de primaria. Revista PULSO, 43, 55-74.

Mayer, J.D., \& Salovey, P. (1997). What us Emotional Intelligence: Implications for Educators. Harper Collins.

Merchán, I. M., Bermejo, M. L., y De Dios, J. (2014). Eficacia de un Programa de Educación Emocional en Educación Primaria. Revista de Estudios e Investigación en Psicología y Educación, 1(1), 91-99.

Ministerio de Educación, Cultura y Deporte (2015). Orden ECD/65/2015, de 21 de enero, por la que se describen las relaciones entre las competencias, los contenidos y los criterios de evaluación de la educación primaria, la educación secundaria obligatoria y el bachillerato. Publicado en Boletín Oficial del Estado no25, del 29 de enero de 2015. España.

Pastor-Arnau, J. (2019). Disseny, aplicació i avaluació d'un programa d'educació emocional a través de la música en $4 \mathrm{t}$ d'educació primària. Revista d'Innovació i Recerca en Educació, 12(1), 1-21.

Pérez, N., y Filella, G. (2019). Educación emocional para el desarrollo de competencias emocionales en niños y adolescentes. Praxis \& Saber, 10(24), 23-44.

Postigo-Zegarra, S., Schoeps, K., Montoya-Castilla, I., y Escartí, A. (2019). Programa de educación emocional para adolescentes (PREDEMA): valoración desde la perspectiva de los participantes y sus efectos en las competencias socio-afectivas. Journal for the Study of Education and Development, 42(2), 303-336.

Ruiz-Aranda, D., Salguero, J., y Cabello, R. (2012). Can an emotional intelligence program improve adolescent's psychosocial adjustment? Results from de INTEMO Project. Social Behavior and Personality, 40(8), 1373-1380.

Schoeps, K., Villanueva, L., Prado-Gascó, V. J. y Montoya-Castilla, I. (2018). Development of Emotional Skills in Adolescents to Prevent Cyberbullying and Improve Subjective Well- Being. Frontiers in Psychology, 9, 1-12.

Sigüeña-Marín, V. S., Carballido-Guisado, R., Pérez-Albéniz, A., y Fonseca-Pedrero, E. (2019). Implementación y evaluación de un programa de inteligencia emocional en adolescentes. Universitas Psychologica, 18(3), 1-13. 
Stage, S.A., y Quiroz, D.R. (1997). A Meta-Analysis of Interventions to Decrease Disruptive Classroom Behavior in Public Education Settings. School Psychology Review, 26(3), 333- 68.

Torres, J. M., y Piscoya, C. A. (2014). Inteligencia emocional y desarrollo de las primeras habilidades sociales en sexto grado. UCV-HACER, Revista de Investigación y Cultura, 3(2), 1-8.

Tur-Porcar, A., Llorca-Mestre, A., y Mestre-Escrivà, V. (2021). Agresividad, inestabilidad y educación socioemocional en un entorno inclusivo. Revista científica de Educomunicación, 66, 4455.

Urrútia, G. y Bonfill, X. (2010). Declaración PRISMA: una propuesta para mejorar la publicación de revisiones sistemáticas y metaanálisis. Medicina clínica, 135(11), 507- 511.

Vivas, M. (2003). La educación emocional: conceptos fundamentales. Sapiens. Revista Universitaria.de Investigación, $4(2), 1-21$.

Wilson, D. B., Gottfredson, D. C., \& Najaka, S. S. (2001). SchoolBased Prevention of Problem Behaviors: A Meta-Analysis. Journal of Quantitative Criminology, 17, 247-272.

Wilson, S.J. y Lipsey, M.W (2007). School-Based Interventions for Aggressive and Disruptive Behavior: Update of a Meta-Analysis. American Journal of Preventive Medicine, 33(2),130-143. 